\title{
Candida and invasive candidiasis: Back to basics.
}

\begin{abstract}
The ubiquitous Candida spp. is an opportunistic fungal pathogen which, despite treatment with antifungal drugs, can cause fatal bloodstream infections (BSIs) in immunocompromised and immunodeficient persons. Thus far, several major $\mathrm{C}$. albicans virulence factors have been relatively well studied, including morphology switching and secreted degradative enzymes. However, the exact mechanism of Candida pathogenesis and the host response to invasion are still not well elucidated. The relatively recent discovery of the quorum-sensing molecule farnesol and the existence of quorum sensing as a basic regulatory phenomenon of the $\mathrm{C}$. albicans population behavior has revolutionized Candida research. Through population density regulation, the quorum-sensing mechanism also controls the cellular morphology of a C. albicans population in response to environmental factors, thereby, effectively placing morphology switching downstream of quorum sensing. Thus, the quorum-sensing phenomenon has been hailed as the 'missing piece' of the pathogenicity puzzle. Here, we review what is known about Candida spp. as the etiological agents of invasive candidiasis and address our current understanding of the quorum-sensing phenomenon in relation to virulence in the host.
\end{abstract}

Keyword: Candida; Candidiasis; Invasive. 